\title{
Evaluating the Safety Impact of Connected and Autonomous Vehicles with Lane Management on Freeway Crash Hotspots Using the Surrogate Safety Assessment Model
}

\author{
Hui Zhang $\mathbb{D}^{1,2}$ Ninghao Hou $\mathbb{D}^{1,2}$ Jianhua Zhang $\mathbb{D}^{1,2,3}$ Xuyi Li $\mathbb{D}^{1,2}$ and Yan Huang $\mathbb{D}^{1,2}$ \\ ${ }^{1}$ Intelligent Transportation Systems Research Center, Wuhan University of Technology, Wuhan 430063, China \\ ${ }^{2}$ Engineering Research Center of Transportation Safety, Wuhan 430063, China \\ ${ }^{3}$ Wuhan Traffic Management Bureau, Wuhan 430030, China
}

Correspondence should be addressed to Jianhua Zhang; zjhls@whut.edu.cn

Received 7 February 2021; Accepted 25 June 2021; Published 7 July 2021

Academic Editor: Yanyong Guo

Copyright (c) 2021 Hui Zhang et al. This is an open access article distributed under the Creative Commons Attribution License, which permits unrestricted use, distribution, and reproduction in any medium, provided the original work is properly cited.

One goal for large-scale deployment of connected and autonomous vehicles is to achieve the traffic safety benefit since connected and autonomous vehicles (CAVs) could reduce the collision risk by enhancing the driver's situation perception ability. Previous studies have analyzed the safety impact of CAVs involved in traffic, but only few studies examined the safety benefits brought by CAVs when approaching high-collision-risk road segments such as the freeway crash hotspots. This study chooses one freeway crash hotspot in Wuhan, China, as an instance and attempts to estimate the safety benefits for differential penetration rates (PRs) of CAVs using the surrogate safety assessment model (SSAM). First, the freeway crash hotspot is identified with kernel density estimation and simulated by VISSIM. Then, the intelligent driver model (IDM) and Wiedemann 99 (a car-following model) are adopted and calibrated to control the driving behaviors of CAVs and human-driven vehicles (HVs) in this study, respectively. The impact that rather CAVs are constrained with or without managed lanes on traffic safety is also discussed, and the PR of CAVs is set from $10 \%$ to $90 \%$. The results of this study show that when the PR of CAVs is lower than $50 \%$, there is no significant improvement on the safety measures such as conflicts, acceleration, and velocity difference, which are extracted from the vehicle trajectory data using SSAM. When the penetration rate is over $70 \%$, the experiment results demonstrate that the traffic flow passing the freeway hotspot is with fewer conflicts, smaller acceleration, and smaller velocity difference in the scenario where CAVs are constrained with managed lane compared with the scenario without managed lane control. The safety benefit that CAVs bring needs to be discussed. The lane management of CAVs will also lead to distinct safety impact.

\section{Introduction}

As vehicle-to-everything (V2X), vehicle sensors, on-board computers, and calculating efficiency develop, more connected and autonomous vehicles (CAVs) will be involved in traffic flow on road [1]. The development and application of technology need time; a mixed condition of CAVs and human-driven vehicles (HVs) will exist for several years [2].

For the benefit of situation perception technology and quick-response driving behavior, the involvement of CAVs would help improve traffic safety levels [3]. But due to the driving behavior difference and decision-making difference between CAV and HVs, CAVs and HVs may disturb each other in the mixed condition, which may lead to hidden troubles on traffic safety [4]. The impact of the involving of CAVs on traffic remains unclear [5-7].

On the one hand, some research studies indicated that the involvement of CAVs might be of benefit to traffic safety. CAVs can improve string stability by preventing shockwaves [8]. CAVs equipped with beyond-line-of-sight ability can significantly improve safety by preventing the cascading of braking events [9]. The increase of penetration rate (PR) of CAVs would greatly improve traffic safety in the mixed flow in the way of keeping time to collision (TTC) an appropriate range [10]. In the mixed condition of CAVs and HVs, as the PR of CAVs increases, the potential conflicts decline both in 
intersections and highways [11]. A CAV control algorithm improves road safety significantly by reducing conflicts even at low PRs of CAVs [12]. Based on crash data of 6 countries from 2012 to 2016, the reduction of the average number of crashes by $47.48 \%$ was revealed if all vehicles were equipped with connected vehicle (CV) or autonomous vehicle (AV) technologies [13]. These research studies demonstrated the advantages of CAVs in traffic safety. On the other hand, some researchers demonstrated that the mixed condition would cause interference between CAVs and HVs and negatively affect traffic safety under certain conditions $[4,14]$. The involvement of CAVs may lead to an increase in potential collisions with low PRs of CAVs [11].

Considering the feature of CAVs, a road without lateral interference would make full use of CAVs' capability and improve traffic efficiency and safety with the formation of the fleet. To better investigate the influence of mixed conditions of CAVs and HVs on traffic safety, considering the characteristics of crash hotspots, urban freeway crash hotspots are chosen as the experiment conditions. Research on CAV traffic safety mainly used microsimulation to experiment and obtain data [10,15-18]. To investigate the impact of CAVs and lane management strategy on traffic safety, microsimulation is used $[19,20]$.

Vehicle trajectory data are often used for the analysis of traffic flow characteristics [21]. Conflicts can be measured with trajectory data through the surrogate safety assessment model (SSAM) [22-26]. Combined with microscopic traffic simulation, precise trajectory data can be obtained.

In summary, to investigate the impact of CAVs on traffic safety on urban freeway, a simulation platform is necessary. With a simulation platform, the adjustment of PRs of CAVs is accessible, which could contribute to the further research into the traffic safety impact.

This study advances the understanding of the traffic safety impacts of CAVs on urban freeway crash hotspots with microscopic traffic simulation and explores the proper lane management under different PRs of CAVs. This paper is organized as follows: Section 2 introduces the experiment framework used in this research, which includes the selection of experiment road section, simulation scenarios design, the detail of the simulation and vehicle control algorithms for CAVs and HVs, surrogate safety assessment model for potential conflict detection, and the methods for traffic safety assessment. Section 3 contains the results obtained from the microsimulation and the analysis of the impact of CAVs on traffic safety through the calculation of 3 parameters. Section 4 discusses the limitation of this study and proposes improvement for the future traffic safety research involving CAVs. Section 5 includes the conclusion obtained from the research results.

\section{Methods}

2.1. Experimental Road Segment: Crash Hotspot Site. Crash data used for hotspots identification and analysis are extracted from the Crash Report System developed by the Ministry of Public Security. For each traffic crash case, its detailed crash information was recorded, as shown in Table 1. The crashes that occurred from January 2016 to November 2019 and along the 3rd Ring Road of Wuhan city were used in this study. In summary, 11,498 crashes occurred in this six-lane two-way separated freeway in the period of 47 months.

A kernel density estimation method [27] is used to determine the hotspots in this study, and four crash hotspots are identified and indicated in Table 2 and Figure 1. The fourth hotspot is with the highest kernel density and chosen as the test site, which is located in the section of Luoshi South Road to Qingling Highway Interchange.

Then, a microscopic simulated road is built using VISSIM based on the fourth accident hotspot. This section of the road is 4.6 kilometers long, and it goes east-west with 3 lanes on each direction. The satellite imagery with accident stamps is shown in Figure 2.

In addition to the crash data, the traffic flow data recorded by microwave detectors could be used to represent the traffic condition and as an input of the simulation, as listed in Table 3.

\subsection{Scenarios of CAVs Controlled by Lane Management.} Based on the real road condition of the section of Luoshi South Road to Qingling Highway Interchange on the Wuhan $3^{\text {rd }}$ Ring Road, two scenarios are set. The purpose of these two different microscopic traffic simulation scenarios is to test how lane change management would affect traffic safety with different PRs of CAVs involved. With the setting of various penetration rates, the impact of CAVs on traffic safety could be reflected by conflicts [28, 29].

In scenario 1 , no limits are set to the CAVs that CAVs could change lanes as they want. In scenario 2, a managed lane is set for CAVs where CAVs could only run on this managed lane.

The 4.6-kilometer road is divided into 2 parts: the first 500 meters of it is set to be the preparing part which helps make the traffic flow stable, and the rest part is set as the test part where the data are collected, presented in Figure 3.

For the decision-making mechanisms of CAVs and HVs are different, the response of two different types of vehicles under the same traffic condition would be different.

As time goes by, with the development of technology, the PR of CAVs would keep rising. A proper traffic management strategy of CAVs would be necessary to avoid the mutual interference of driving behaviors that CAVs and HVs would have on each other.

2.2.1. Scenario 1: CAVs Allowed to Change Lanes. As no lane-changing limits are set for the CAVs in scenario 1, a mixed condition of CAVs and HVs is shown as in Figure 4.

2.2.2. Scenario 2: CAVs Constrained to Managed Lanes. CAVs are only allowed to operate on the managed lane, while HVs could operate anywhere on the road at will. Scenario 2 is shown in Figure 5. 
TABle 1: Crash information recorded in the Crash Report System.

\begin{tabular}{lcc}
\hline Parameter & Notes & Sample \\
\hline Time & The time that crash occurred & $2018 / 9 / 320: 07: 00$ \\
Description & The situation description of the crash & Two buses side impact \\
Location & The road name where crash occurred & Wuhan $3^{\text {rd }}$ Ring Road \\
Mileage & The mileage where crash occurred & $K 1+200$ meter \\
Direction & The direction where crash occurred (downstream, upstream) & Downstream \\
\hline
\end{tabular}

TABle 2: The location of 4 crash hotspots.

\begin{tabular}{lc}
\hline Crash hotspots & Location (all four hotspots are on Wuhan $3^{\text {rd }}$ Ring Road) \\
\hline 1 & The section south of Changfeng Bridge \\
3 & The section of Wangjiazui Highway Interchange \\
4 & The section of Yingwu Highway Interchange \\
\hline
\end{tabular}

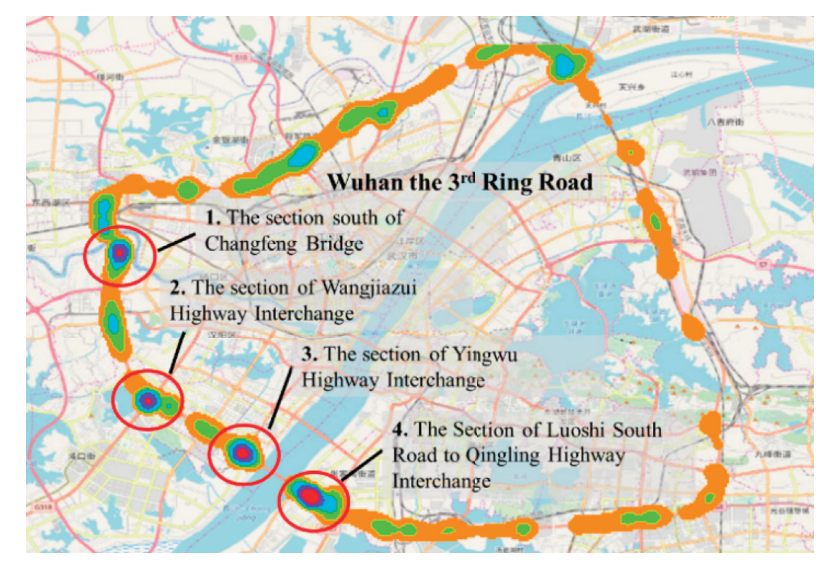

FIgURE 1: Visualization of the spatial distribution of accident density by kernel density estimation.

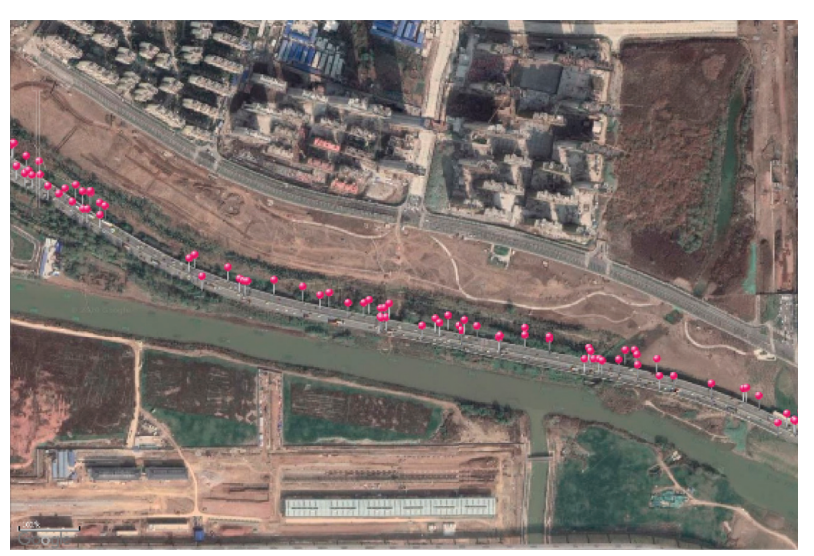

FIgure 2: The location of accidents on the section of Wuhan $3^{\text {rd }}$ Ring Road from Luoshi South Road to Qingling Highway Interchange.

2.3. Microsimulation and Vehicle Control Algorithm. The simulation test is conducted using the microsimulation software VISSIM. The trajectory file is generated from the simulation for surrogate safety measures. Control algorithms for CAVs and HVs shall be calibrated before the simulation.
Two scenarios are built for the simulation. The traffic vehicle composition settings, vehicle input, expected speed, traffic flow, and vehicle proportions are all based on the actual collected traffic flow data collected by microwave detectors as input.

2.3.1. Microsimulation. As for the simulation, to ensure the reliability of the simulation results of 2 scenarios, 5 different random seeds are set for each group of simulations in each scenario. For each scenario, simulations are set 5 times at each PR of CAVs with different random seeds, and the PR of CAVs is in the range of $10 \%$ to $90 \%$ at $10 \%$ interval. Overall, for each scenario, the total number of simulations is 45 .

A single complete simulation consists of two phases, which are warm-up phase and data-recording phase. The set of warm-up phase is to make sure the traffic flow stabilizes during the simulation. Only the data recorded during the data-recording phase are used in data processing. The length of warm-up phase is $3600 \mathrm{~s}$, the length of data-recording phase is $7200 \mathrm{~s}$, and the total time of a complete simulation is $10800 \mathrm{~s}$. All the trajectory files are imported into SSAM to analyze traffic conflicts.

2.3.2. Car-Following Behavior. In our study, with regard to the HV, the Wiedemann 99 model [30] is used to simulate the driving behavior of vehicles on the freeway. And the intelligent driving model (IDM) is adopted for CAV simulation.

For Wiedemann 99 model, the driving behavior is based on the following algorithm, as illustrated in equation (1). And the parameters involved in Wiedemann 99 are listed in Table 4.

$$
\mathrm{d} x_{-} \text {safe }=\mathrm{CC}_{0}+\mathrm{CC}_{1} \cdot v,
$$

where $\mathrm{d} x_{-}$safe denotes the safety distance between the leading vehicle and the following vehicle, $\mathrm{CC}_{0}$ is the average distance when the vehicle is stopped, $\mathrm{CC}_{1}$ denotes the desired headway, and $v$ is the velocity.

The IDM was proposed by Treiber [31] in 2000 and has been used in many pieces of research on the control algorithm of CAVs [28, 32]. 
TABLE 3: Example of the traffic flow data recorded by microwave detectors.

\begin{tabular}{|c|c|c|c|c|c|}
\hline Vehicle type (1-car, 2-truck) & Time & Checkpoint & Direction (south to north) & Velocity $(\mathrm{km} / \mathrm{h})$ & Lane \\
\hline 2 & 20190302230647300 & 1 & South to north & 37 & 3 \\
\hline 2 & 20190303074851300 & 1 & South to north & 46 & 2 \\
\hline 2 & 20190303095717700 & 1 & South to north & 50 & 3 \\
\hline 2 & 20190304005344800 & 1 & South to north & 53 & 3 \\
\hline 2 & 20190304181204800 & 1 & South to north & 53 & 2 \\
\hline 2 & 20190306041031900 & 1 & South to north & 36 & 2 \\
\hline 2 & 20190306132039000 & 1 & South to north & 38 & 2 \\
\hline 2 & 20190306220631700 & 1 & South to north & 32 & 2 \\
\hline 2 & 20190307010854000 & 1 & South to north & 62 & 3 \\
\hline 2 & 20190307024558500 & 1 & South to north & 56 & 2 \\
\hline 2 & 20190307050453600 & 1 & South to north & 67 & 2 \\
\hline 2 & 20190307114304200 & 1 & South to north & 54 & 3 \\
\hline
\end{tabular}

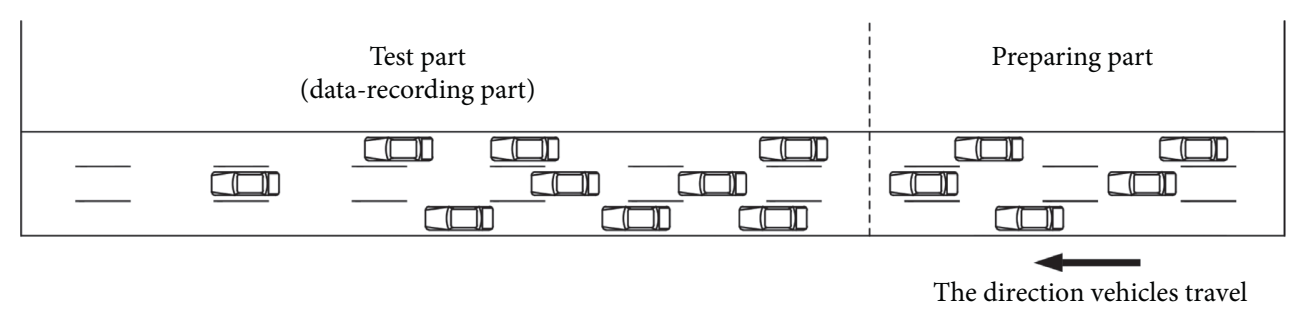

Figure 3: Schematic diagram of the experiment road section.

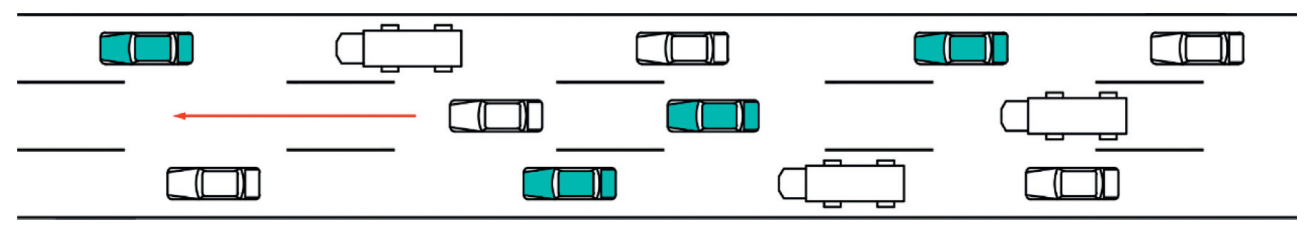

Human-driven vehicle

प10 - CAV

- Tـــ Truck

पD - CAR

FIgURE 4: No lane-changing limits are set for CAVs.

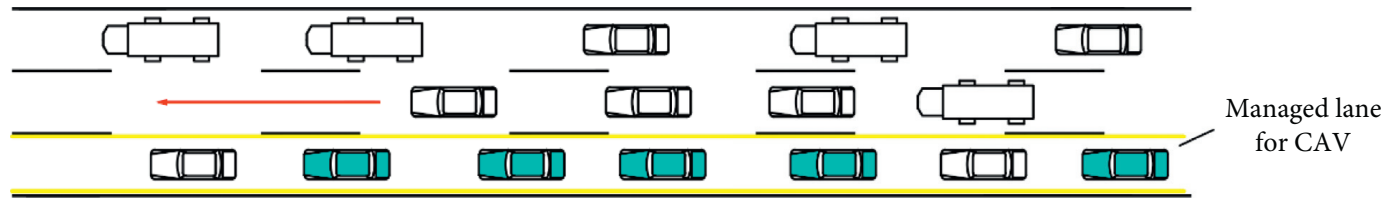

Human-driven vehicle

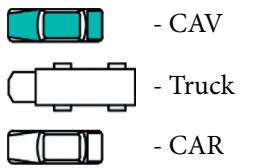

Figure 5: A managed lane is set for CAVs.

The basis of this theoretical car-following model is that assumes the IDM would fit well under both free flow and congested flow. Vehicles controlled by IDM could adjust the head spacing and their velocity to the vehicles ahead. With better stability of vehicle control, the IDM is now wildly used.

As parameters of IDM have clear physical meanings, which could influence driving behaviors of controlled 
TABle 4: Wiedemann 99 parameters.

\begin{tabular}{lc}
\hline Parameter (unit) & Short description \\
\hline $\mathrm{CC}_{0}(\mathrm{~m})$ & Standstill gap \\
$\mathrm{CC}_{1}(\mathrm{~m})$ & Headway time \\
$\mathrm{CC}_{2}(\mathrm{~m})$ & "Following" variation \\
$\mathrm{CC}_{3}(\mathrm{~s})$ & Threshold for entering "following" \\
$\mathrm{CC}_{4}(\mathrm{~m} / \mathrm{s})$ & Negative "following" threshold \\
$\mathrm{CC}_{5}(\mathrm{~m} / \mathrm{s})$ & Positive "following" threshold \\
$\mathrm{CC}_{6}\left(10^{-4} \mathrm{rad} / \mathrm{s}\right)$ & Speed dependency of oscillation \\
$\mathrm{CC}_{7}\left(\mathrm{~m} / \mathrm{s}^{2}\right)$ & Oscillation acceleration \\
$\mathrm{CC}_{8}\left(\mathrm{~m} / \mathrm{s}^{2}\right)$ & Standstill acceleration \\
$\mathrm{CC}_{9}\left(\mathrm{~m} / \mathrm{s}^{2}\right)$ & Acceleration at $80 \mathrm{~km} / \mathrm{h}$ \\
$\mathrm{VDES}$ & Desired speed of vehicles \\
\hline
\end{tabular}

vehicles. Moreover, the model could simultaneously describe the car-following behavior of vehicles on a single lane in the state of both free flow and congested flow. In the state of congested flow, when the velocity difference of two vehicles one after another is small, a slight change of space ahead would not cause rapid deceleration of vehicles. A relatively stable vehicle operating state helps provide a comfortable driving experience and improve traffic safety. The function of IDM is expressed as equations (2) and (3):

$$
\begin{aligned}
a & =a_{0}\left(1-\left(\frac{v}{v_{0}}\right)^{\delta}-\left(\frac{s^{*}(v, \Delta v)^{2}}{s}\right)^{2}\right), \\
s^{*}(v, \Delta v) & =s_{0}+v T+\frac{v \Delta v}{2 \sqrt{a_{0} b}},
\end{aligned}
$$

where $a$ is the acceleration of vehicle $n, v$ is the velocity of vehicle $n, \Delta v$ is the velocity difference of the following vehicle and the vehicle ahead, $v_{0}$ is the expected velocity of the vehicle in the free flow, $a_{0}$ is the acceleration, $b$ is the deceleration, $s_{0}$ is the minimum space headway of the following vehicle when the vehicle stops, $T$ is the expected time headway, and $\delta$ is the parameter which is normally 4.

For an accurate simulation of CAVs, an operation strategy is made. In the free flow, CAVs are expected to pass through the road section at a high speed. In a congested flow, CAVs could keep a shorter space headway. According to previous research [33], there is a reduction coefficient under each traffic condition, as shown in Table 5.

Combining previous research results and traffic characteristics of freeways in China, the parameters and value ranges of the selected IDM are shown in Table 6.

2.3.3. Lane-Changing Behavior. In this research, the lateral movement behaviors of all vehicles in the microsimulation are set to VISSIM default lane-changing control strategy.

2.4. Surrogate Safety Assessment Model. The surrogate safety assessment model is a model developed by the Federal Highway Administration (FHWA), U.S. Department of
TABLE 5: Reduction coefficient of IDM under different traffic conditions.

\begin{tabular}{lllll}
\hline Reduction coefficient & $\eta_{T}$ & $\eta_{v_{0}}$ & $\eta_{a_{0}}$ & $\eta_{b}$ \\
\hline Smooth traffic flow & 1.0 & 1.0 & 1.0 & 1.0 \\
Congestion & 0.5 & 0.9 & 1.0 & 1.0 \\
\hline
\end{tabular}

TABLE 6: The range of parameters of IDM.

\begin{tabular}{lccccc}
\hline Parameter & $v_{0}$ & $a_{0}$ & $b$ & $T$ & $s_{0}$ \\
\hline Upper bound & 80 & 0.1 & 0.1 & 0.1 & 0.1 \\
Lower bound & 100 & 5 & 5 & 5 & 10 \\
Value & 86 & 1.2628 & 2.6907 & 1.5295 & 7.8893 \\
\hline
\end{tabular}

Transportation, to automatically identify, classify, and evaluate potential vehicle-to-vehicle conflicts with trajectory files. A trajectory file could be output after running the microsimulation on VISSIM, which contains data about the positions, velocities, and other data of vehicles. SSAM uses several algorithms to identify potential conflicts based on vehicle trajectory files. Software with SSAM built in is developed by the FHWA on Windows.

Several parameters can be adjusted to determine the criterion of identifying conflicts. The criterion of conflicts detecting could be affected by the change of several parameters.

TTC is the minimum time-to-collision value during the conflict [34]. A TTC is the time step between the identification moment to the collision moment. As TTC is set larger, more potential conflicts will be detected.

To classify different types of conflicts, the conflict angle is set. A conflict angle is the approximate angle of the conflicting vehicles in a potential collision.

According to the conflict angle, vehicle-to-vehicle conflicts are divided into 3 types, presented in Figure 6. If $\theta$ is smaller than $\theta_{1}$, the conflict is of rear-end conflict. If $\theta$ is bigger than $\theta_{2}$, the conflict is of crossing conflict. If $\theta$ is bigger than $\theta_{1}$ and smaller than $\theta_{1}$, the conflict is of lanechange conflict.

2.5. Assessment of Traffic Safety. To assess the impact of CAVs on traffic safety, three methods are used, which are the number of conflicts, acceleration distribution, and velocity. All these analyzing data are based on the data output from simulation.

2.5.1. Number of Conflicts. Conflicts between vehicles could result in crash; therefore, the number of conflicts could represent the performance of traffic safety [35]. The greater the number of conflicts, the lower the level of traffic safety.

2.5.2. Acceleration Distribution. Acceleration distribution of vehicles could represent the stability of traffic flows of the road section [36]. As crash occurs, the distance between vehicles is zero. In the process vehicles getting close to each other, a rapid acceleration or deceleration would occur. The 

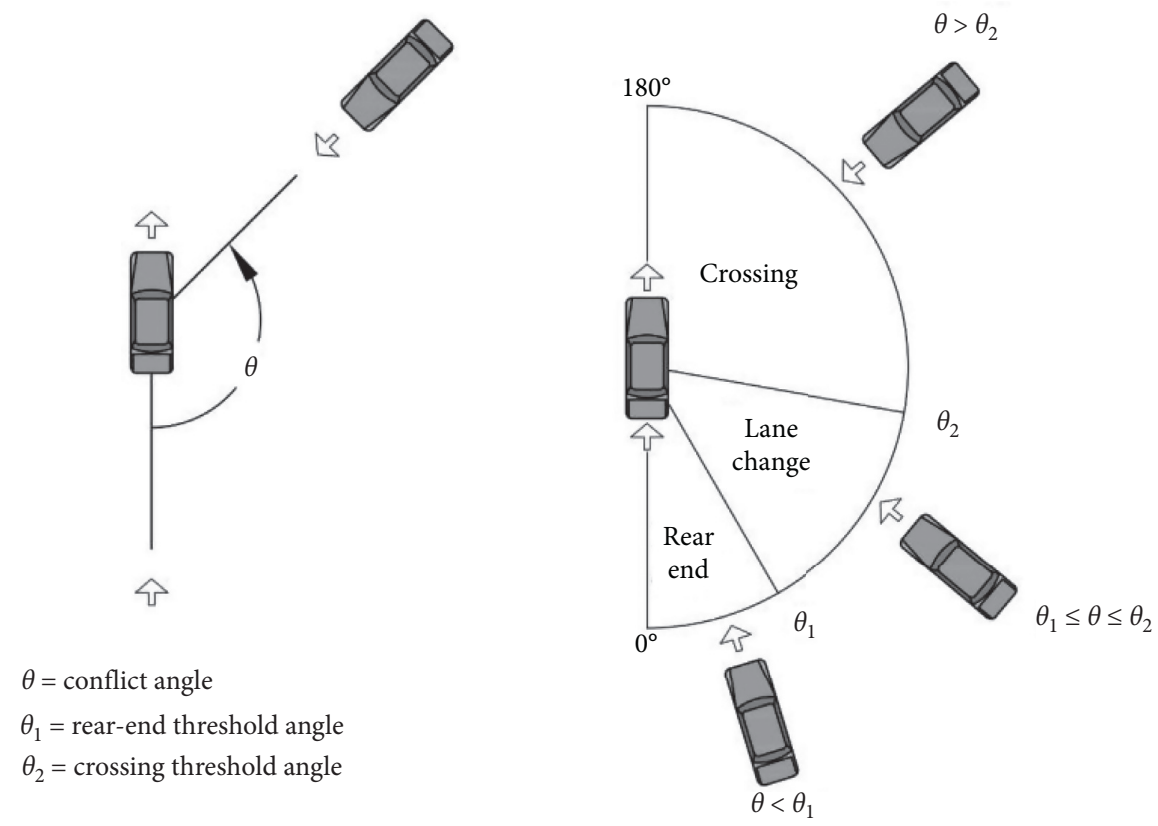

FIgURE 6: Diagrammatic sketch of conflict angle (source: [34]).

occurrence of a crash must be accompanied by intense acceleration or deceleration. Acceleration can indicate road safety [10]. As the acceleration closes to zero, the state of vehicles in traffic flow tends to keep stable, which represents a high level of traffic safety.

2.5.3. Velocity Difference. The distribution of velocity difference can represent the fluctuation of traffic flow. The velocity difference is the difference value between the velocity of front vehicle and the velocity of rear vehicle. As the absolute value of velocity difference gets higher, the distance between vehicles gets further or closer, which declares an unstable state of traffic flow.

The velocity difference is expressed as follows:

$$
v_{\text {diff }}=v_{\text {front }}-v_{\text {rear }},
$$

where $v_{\text {diff }}$ is the velocity difference, $v_{\text {front }}$ is the velocity of front vehicle, and $v_{\text {rear }}$ is the velocity of rear vehicle.

\section{Results}

The results obtained from the microsimulation are divided into 3 parts. Two different scenarios were tested in the microsimulation, and these results are measured as the mean value of the simulation output of all 5 random seeds. These results are the parts after the simulation has been started at $3600 \mathrm{~s}$ when the vehicle flows tended to remain relatively stable. The conflicts are exported from trajectory files analyzed through SSAM. With the existence of medial divider, considering only traffic flows in the same direction, no crossing conflict would exist, conflicts between vehicles are in the type of rear end and lane change regarding the conflict angle of two cars. All the parameters are set to default in SSAM.
3.1. Conflicts on Urban Freeway. Two scenarios are set for the microscopic simulation experiment.

In the simulation, the only parameter that has been changed is the PR of CAVs. The conclusion obtained in the simulation test is that while all the vehicles on road are CAVs, there would not be potential conflicts.

In scenario 1, no managed lane is set for CAVs; all the vehicles could change lanes or overtake other vehicles. The trend of changes in the number of potential conflicts is shown in Figure 7. The number of potential conflicts with various PRs of CAVs is listed in Table 7. As the PR of CAVs increases from $10 \%$ to $50 \%$, the number of potential conflicts increases from 179 to 724 , an increase of $304.47 \%$ on the number of conflicts at a PR of $10 \%$. This indicates that the involvement of CAVs interferes with the running status of traffic flow and lowers the traffic safety level. From $50 \%$ to $80 \%$ of PR of CAVs, the number of potential conflicts barely changes. The mixed condition of CAVs and HVs tends to be stable. When the PR of CAV changes from $80 \%$ to $90 \%$, the number of potential conflicts decreases from 726 to 584 , a decrease of $19.56 \%$ on the number of conflicts at a PR of $80 \%$. As CAVs take a large proportion of traffic flow, the advantage of CAVs starts to reveal.

In scenario 2, managed lanes are set to constrain lanechange behaviors of CAVs. Figure 8 provides a visual representation of the trend of the number of potential conflicts with the increase of PRs of CAVs. As the PR of CAVs increases from $10 \%$ to $90 \%$, the number of potential conflicts decreases from 63 to 0 , a decrease of $100 \%$ on the number of conflicts at a PR of $10 \%$. The results indicate that setting managed lanes and constraining lane-change behaviors help CAVs avoid lateral interference and form fleet. CAV fleet could operate fast and smoothly. In the CAV fleet, CAVs could keep a short head spacing between each other. 


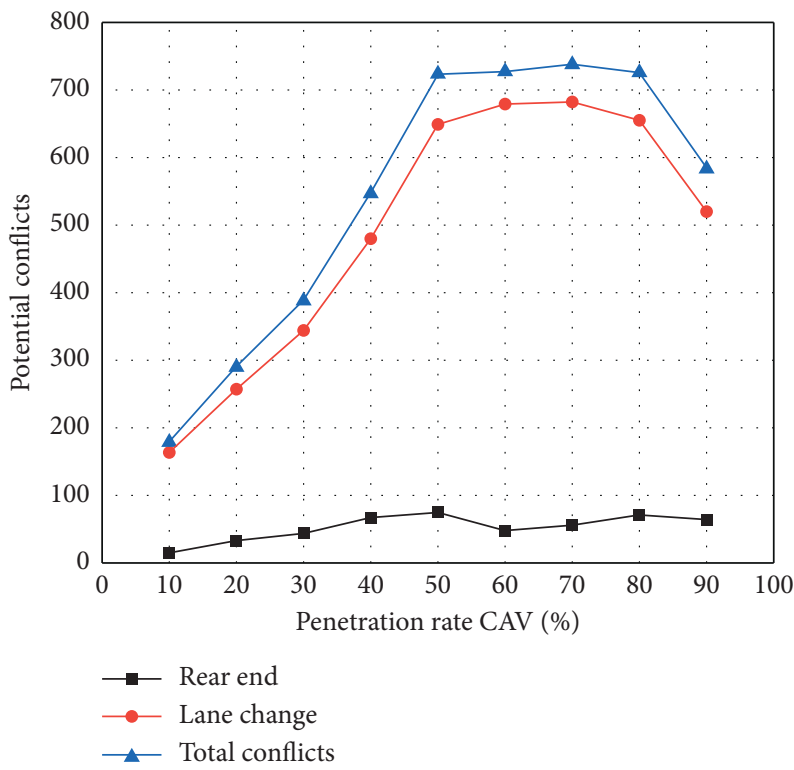

Figure 7: Number of potential conflicts in scenario 1 in $10 \%$ increments of CAV penetration rate.

TABLE 7: Tabular representation of the number of potential conflicts in two scenarios.

\begin{tabular}{|c|c|c|c|c|c|c|}
\hline \multirow{2}{*}{ CAV penetration rate $(\%)$} & \multicolumn{3}{|c|}{ Scenario 1} & \multicolumn{3}{|c|}{ Scenario 2} \\
\hline & Rear end & Lane change & Total conflicts & Rear end & Lane change & Total conflicts \\
\hline 10 & 15 & 164 & 179 & 8 & 55 & 63 \\
\hline 20 & 33 & 257 & 290 & 12 & 45 & 57 \\
\hline 30 & 44 & 344 & 388 & 21 & 24 & 45 \\
\hline 40 & 67 & 480 & 547 & 6 & 7 & 13 \\
\hline 50 & 75 & 649 & 724 & 3 & 4 & 7 \\
\hline 60 & 48 & 679 & 727 & 0 & 4 & 4 \\
\hline 70 & 56 & 682 & 738 & 0 & 2 & 2 \\
\hline 80 & 71 & 655 & 726 & 0 & 0 & 0 \\
\hline 90 & 64 & 520 & 584 & 0 & 0 & 0 \\
\hline
\end{tabular}

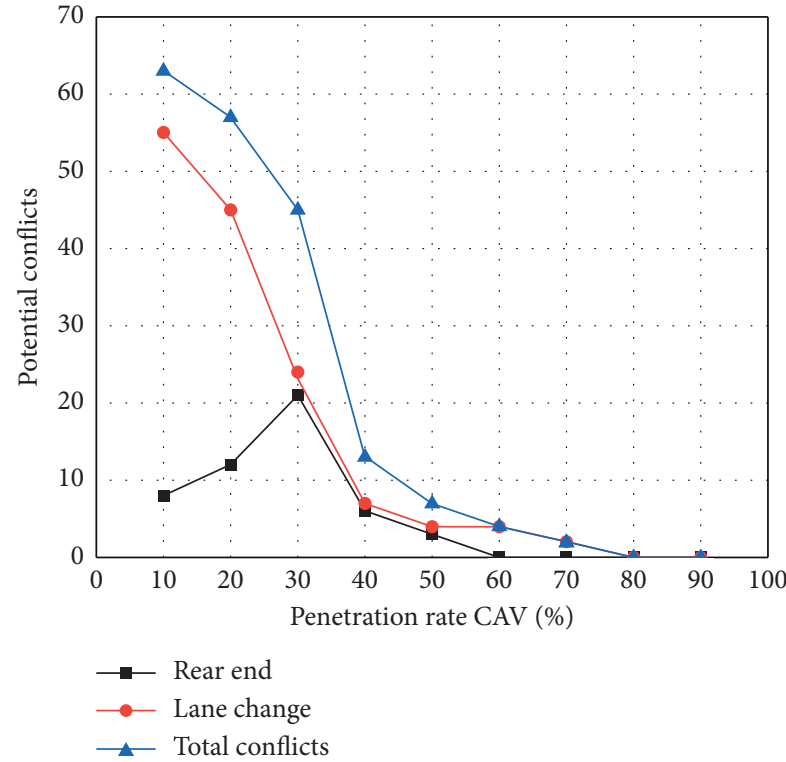

Figure 8: Number of potential conflicts in scenario 2 in $10 \%$ increments of CAV penetration rate.
3.2. Acceleration Distribution on Urban Freeway. Figure 9 presents acceleration distributions of two scenarios with the PR of CAVs increases from $10 \%$ to $90 \%$. Figure 10 provides a visual representation of the change in standard deviation (SD) of acceleration as the PR of CAVs changes. The SD of acceleration and PR are listed in Table 8 .

As Figure 9 shows, under both scenarios, a gradual increase in the ratio of the acceleration at $0 \mathrm{~m} / \mathrm{s}^{2}$ can be easily found, which indicates that the involvement of CAVs would boost the portion of smooth driving in the mixed traffic flow. With the increase in the PR of CAVs, the ratio of high deceleration rate is also decreased, which indicates that a smoother traffic flow can be attained.

3.3. Velocity Difference on Urban Freeway. According to the data of the microwave detector, traffic conditions on road can be analyzed. Comparing data recorded by the microwave detector and data exported through the simulation, the impact that CAVs made can be detected. Figure $11 \mathrm{dem}-$ onstrates the velocity difference of vehicles in real situations and human-driven vehicles $(\mathrm{PR}$ of $\mathrm{CAVs}=0)$ in simulation. The distribution of velocity difference is close to a normal 

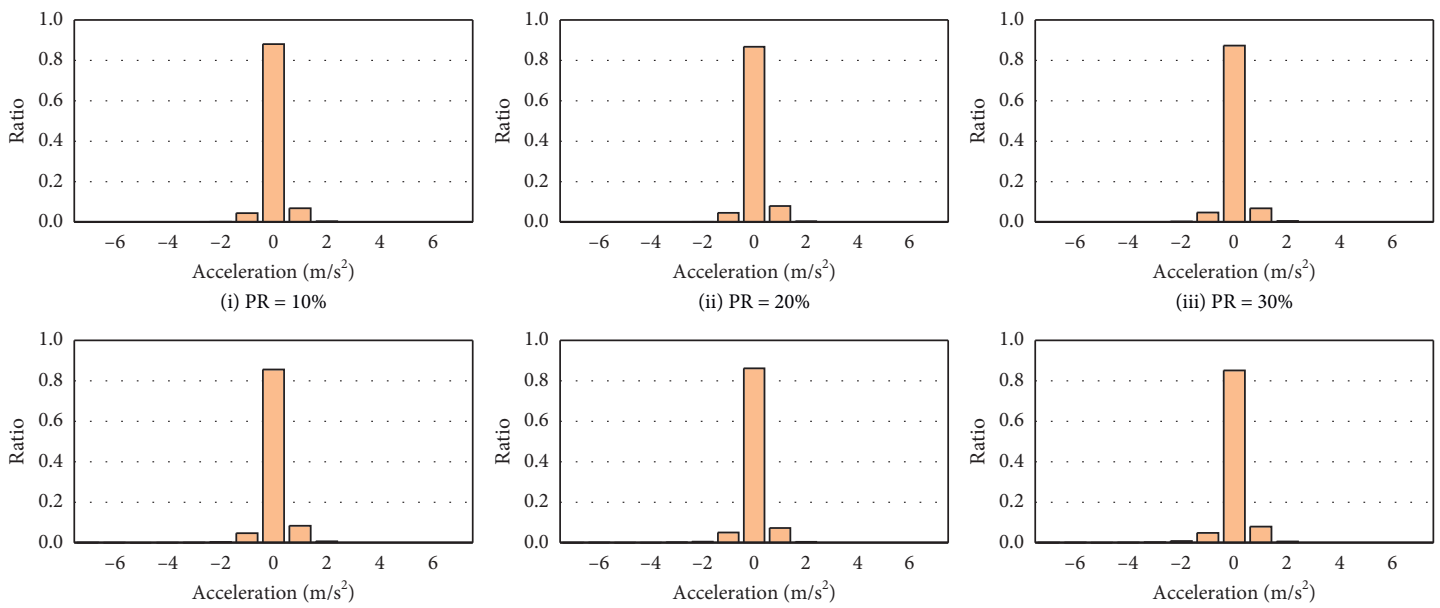

(v) $\mathrm{PR}=50 \%$
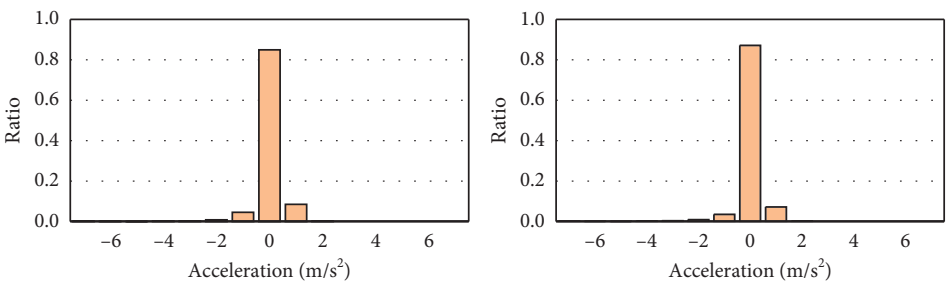

(vi) $\mathrm{PR}=60 \%$

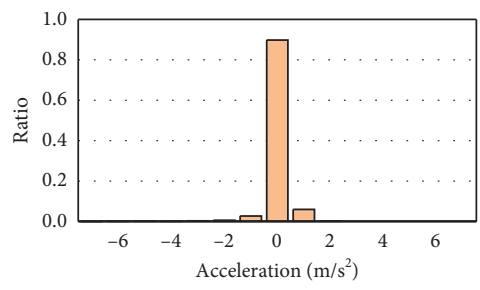

(a)
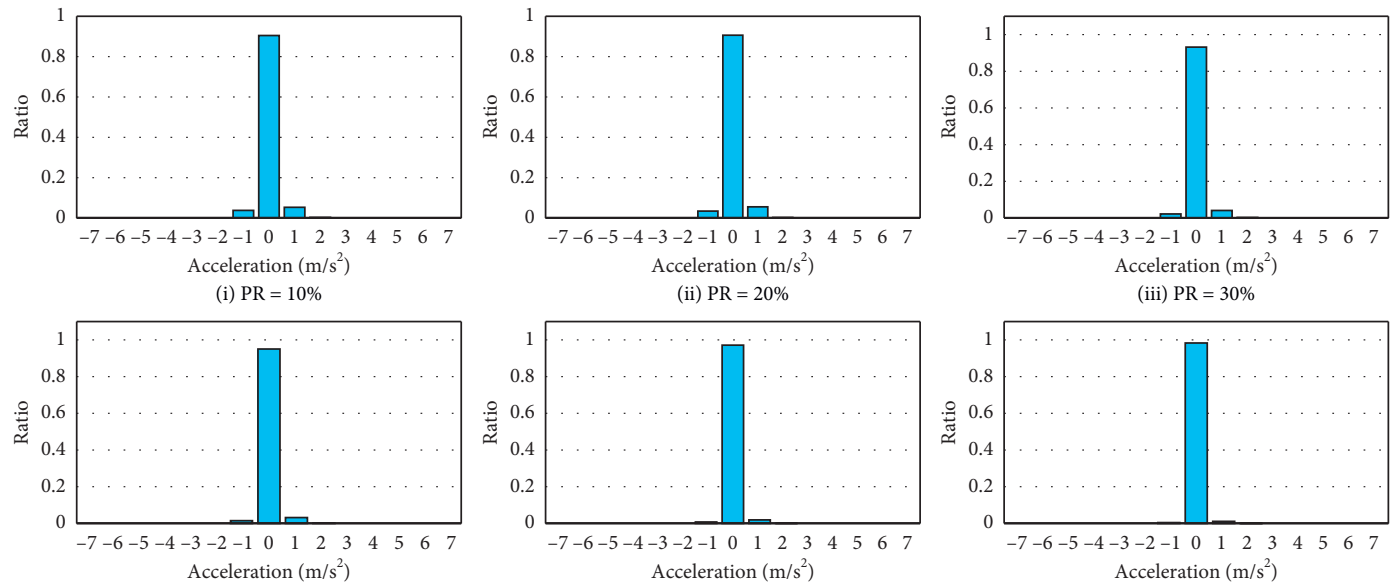

(iii) $\mathrm{PR}=30 \%$

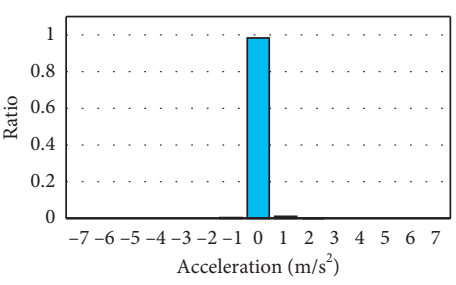

(v) $\mathrm{PR}=50 \%$
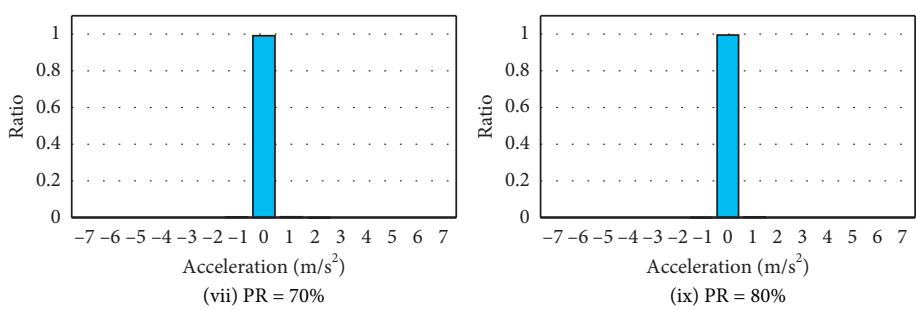

(vi) $\mathrm{PR}=60 \%$

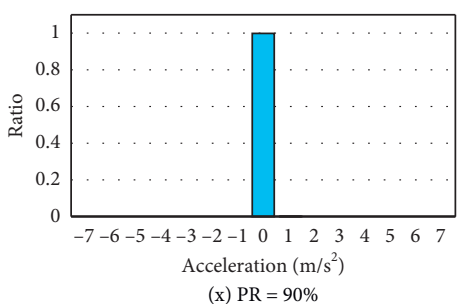

(b)

Figure 9: Acceleration of vehicles on the road of 2 scenarios at different PRs. (a) Scenario 1. (b) Scenario 2.

distribution. The SD of velocity difference on road is 10.504 , and the SD of velocity difference in simulation is 8.653 . Despite little difference, the close SD indicates the accuracy of simulation is high.

Previous studies showed that CAVs can affect traffic safety by affecting the speed of the front and rear cars [10]. In this research, vehicle velocity differences have been calculated under various PRs of CAVs from $10 \%$ to $90 \%$. Figure 12 presents the distribution of velocity differences of vehicles on the road of 2 scenarios. As shown in Figure 12, the velocity difference distribution is like normal distribution. In the mixed traffic flow, as the PR of CAVs increases, 


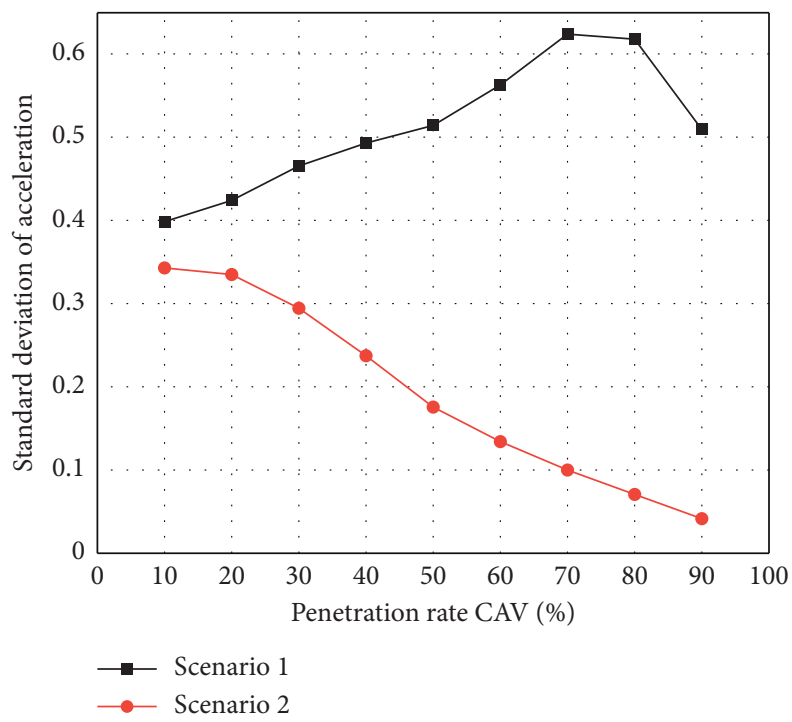

FIGURE 10: Standard deviation distribution of acceleration of vehicles in simulation.

TABLE 8: Tabular representation of the standard deviation of acceleration in two scenarios.

\begin{tabular}{lcc}
\hline PR of CAVs (\%) & SD of acceleration & Scenario 2 \\
\hline 10 & Scenario 1 & 0.34323 \\
20 & 0.39832 & 0.33488 \\
30 & 0.42410 & 0.29471 \\
40 & 0.46552 & 0.23735 \\
50 & 0.49242 & 0.17574 \\
60 & 0.51403 & 0.13405 \\
70 & 0.56258 & 0.09934 \\
80 & 0.62391 & 0.07070 \\
90 & 0.61760 & 0.04140 \\
\hline
\end{tabular}

velocity differences tend to close to 0 , which represents that vehicles on road keep a relatively stable flow. These results show that CAVs can promote the stability of traffic flow. In scenario 2, the velocity differences converge to 0 faster than those in scenario 1, indicating that CAVs could help improve traffic safety on freeway crash hotspots.

As shown in Table 9, as the PR of CAVs increases, the SD of velocity difference in scenario 1 rises and then decreases. The trend of SD of velocity difference in scenario 2 falls, follows with rise, and then falls. But the range of SD of velocity difference is smaller in scenario 2 than in scenario 1 .

Standard deviation is a good indicator to describe the degree of dispersion in a normal distribution. As shown in Figure 13, on the whole, the SD of velocity difference decreases as the PR of CAVs increases.

The velocity difference between the vehicles and the following vehicles can reflect the traffic safety level at the microscope level. Vehicles driving smoothly not only make drivers and passengers feel comfortable but also help increase the volume of traffic flow. In general, CAVs can promote the tendency of the velocity difference between the vehicles and the following vehicles to converge toward 0 . As the PR of CAVs increases, the trend of aggregation of velocity difference is getting more obvious, which could represent that CAVs could help the traffic flow operate smoothly. Constraining CAVs on the managed lane could help improve traffic safety with high PR of CAVs at the freeway crash hotspots. In the condition that CAVs can change lanes freely, velocity difference with different PRs of CAVs is not the same, but on the whole view, there is an aggregate trend that velocity difference gets close to 0 . The reason why there is a trend is that, compared with HVs, CAVs can better perceive traffic conditions ahead and adjust their operation in time to keep a relative safe distance to the vehicle ahead in time. With a more sensitive response in the car-following flow, vehicles (include CAVs) can travel more smoothly and avoid nonsense deceleration and congestion. In this way, setting lane-change constrain to CAVs could better use the strengths of CAVs without the interference of HVs. The biggest feature of driver behaviors is random and unpredictable. With random deceleration, lane changing, and over-passing behaviors, the balance of traffic flow on road can be easily broken. That is one of the reasons why traffic jams would occasionally happen on road. In summary, as the PR of CAVs getting higher, the stability of the overall traffic flow tends to get better, where setting constraints to CAVs' lane-change behavior helps improve traffic safety. 


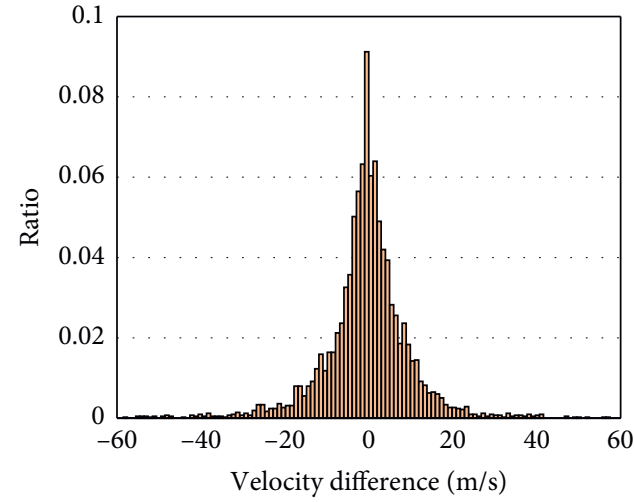

(a)

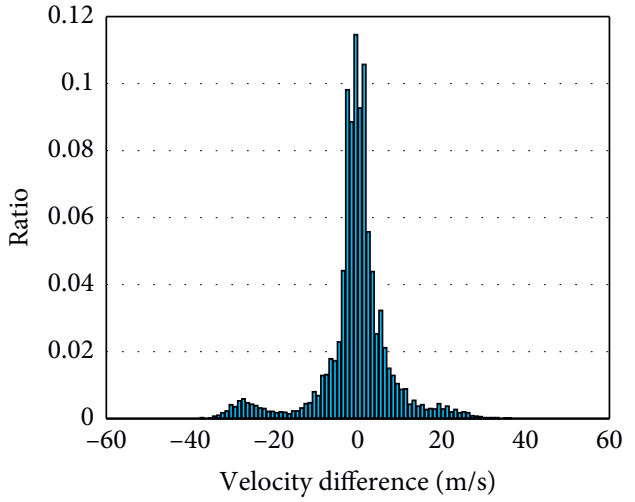

(b)

Figure 11: Velocity difference of vehicles from 7:00 to 9:00 on the selected road section. (a) Velocity difference of vehicles on the road. (b) Velocity difference of HVs in simulation.

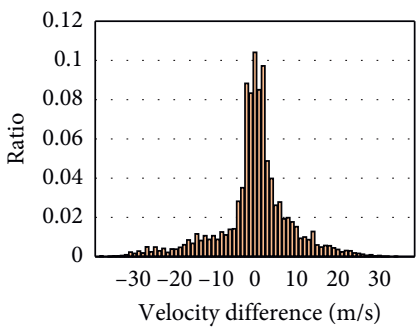

(i) $\mathrm{PR}=10 \%$

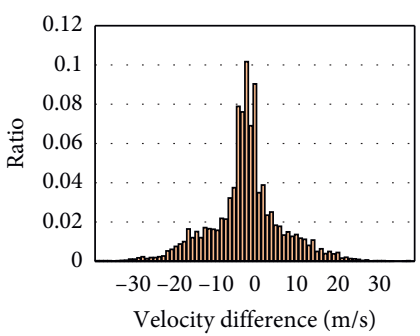

(iv) $\mathrm{PR}=40 \%$

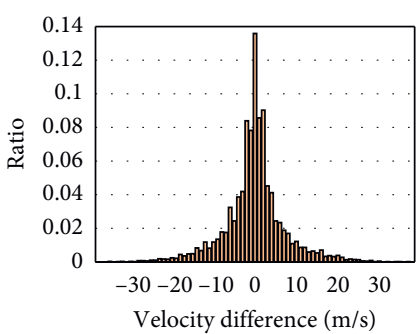

(vii) $\mathrm{PR}=70 \%$

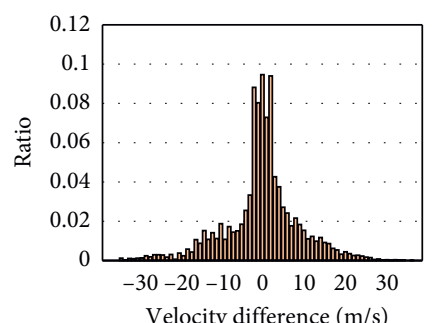

(ii) $\mathrm{PR}=20 \%$

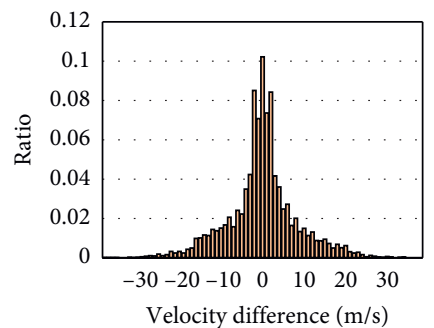

(v) $\mathrm{PR}=50 \%$

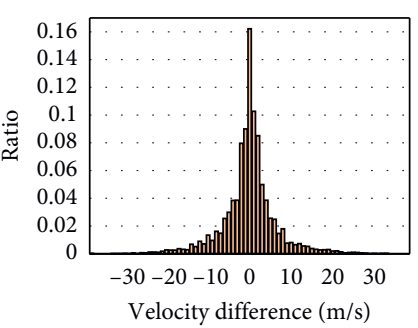

(ix) $\mathrm{PR}=80 \%$

(a)

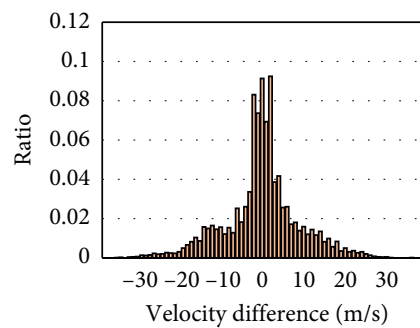

(iii) $\mathrm{PR}=30 \%$

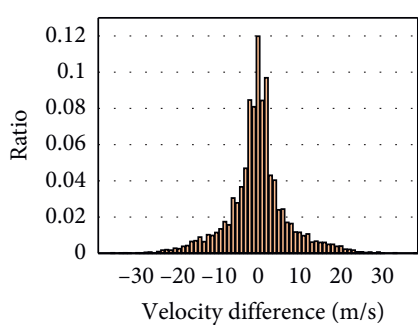

(vi) $\mathrm{PR}=60 \%$

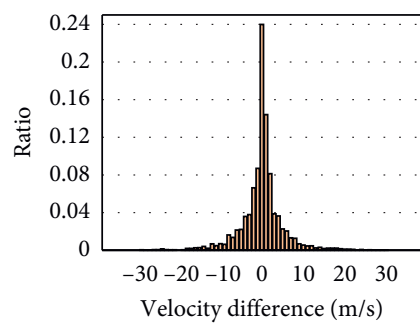

(x) $\mathrm{PR}=90 \%$

FIgURE 12: Continued. 


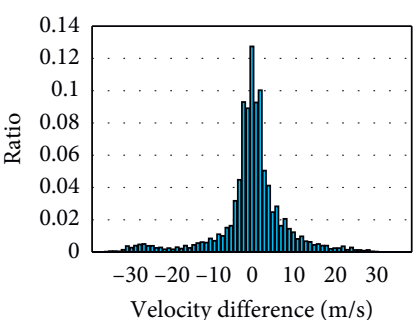

(i) $\mathrm{PR}=10 \%$

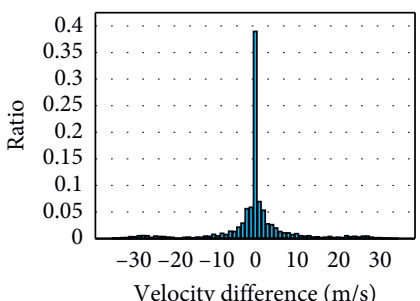

(iv) $\mathrm{PR}=40 \%$

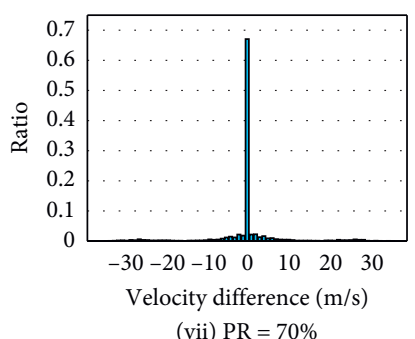

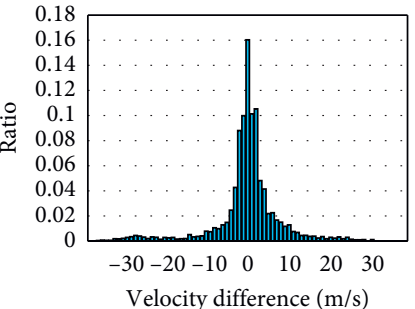

(ii) $\mathrm{PR}=20 \%$

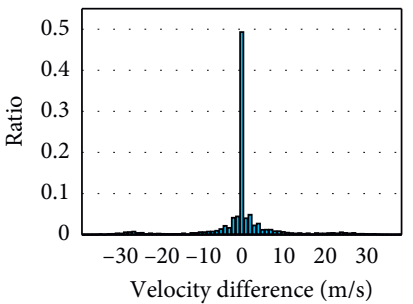

(v) $\mathrm{PR}=50 \%$

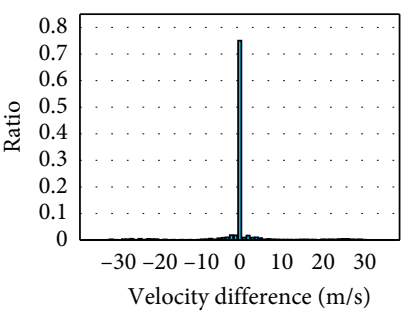

(ix) $\mathrm{PR}=80 \%$

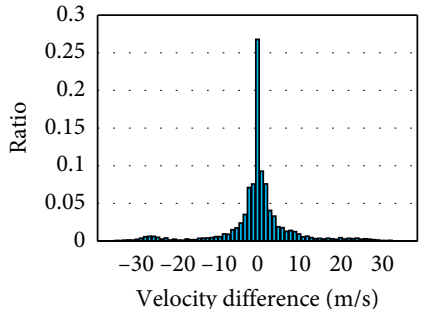

(iii) $\mathrm{PR}=30 \%$

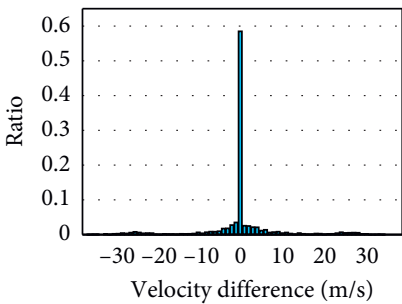

(vi) $\mathrm{PR}=60 \%$

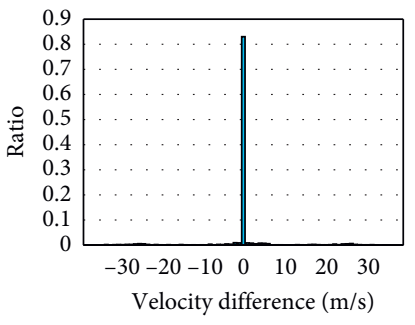

(x) $\mathrm{PR}=90 \%$

(b)

FIGURE 12: Velocity difference of vehicles on the road of 2 scenarios. (a) Scenario 1. (b) Scenario 2.

TABLE 9: Tabular representation of the standard deviation of velocity difference in two scenarios.

\begin{tabular}{lcccccccccc}
\hline SD of velocity difference & $0 \%$ & $10 \%$ & $20 \%$ & $30 \%$ & $40 \%$ & $50 \%$ & $60 \%$ & $70 \%$ & $80 \%$ & $90 \%$ \\
\hline Scenario 1 & 8.653 & 8.752 & 8.914 & 8.918 & 8.688 & 8.335 & 7.269 & 7.226 & 6.449 & 5.287 \\
Scenario 2 & 8.653 & 8.497 & 8.156 & 8.337 & 8.711 & 8.857 & 8.522 & 8.542 & 7.956 & 7.112 \\
\hline
\end{tabular}

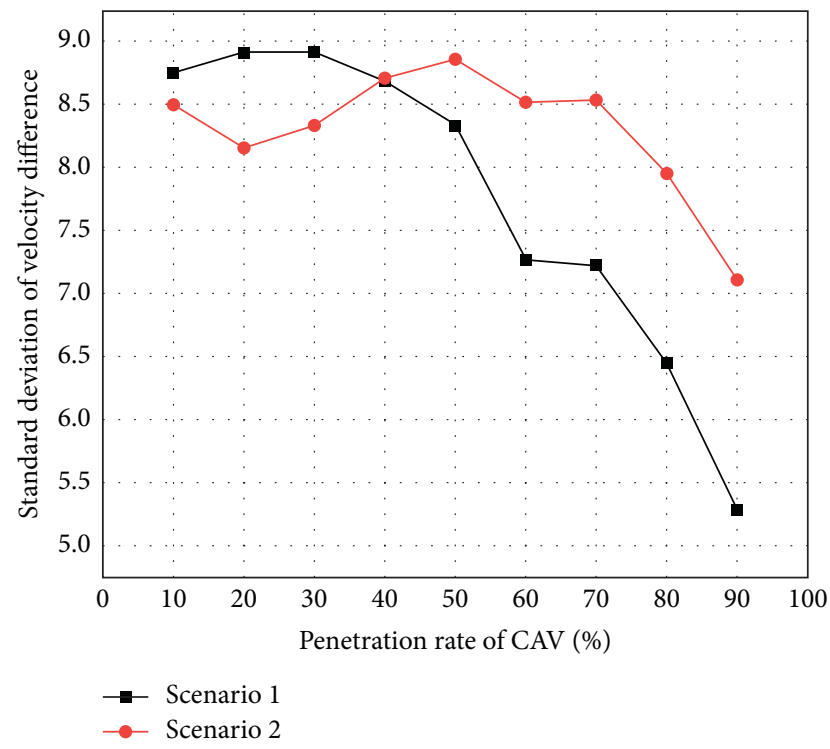

FIgURE 13: The standard deviation of velocity difference of vehicles in simulation. 


\section{Conclusions}

In this research, the impact of CAVs on traffic safety with various PRs of CAVs is investigated by simulation, and two experiment scenarios are set to study the traffic safety improvement of two traffic management strategies.

The authors built a mixed traffic scene with CAVs and HVs using microscopic simulation software. During the experiment, the PR of CAVs has been changed from $10 \%$ to $90 \%$, two traffic management strategies were designed by setting managed lanes for CAVs or not. Evaluation data were generated from the simulation, and potential conflict detection was completed through the surrogate safety assessment model using vehicle trajectory files.

Though that the situation perception advantages seem to provide CAVs with significant improvement in traffic safety, the results indicate that the involvement of CAVs in the mixed traffic flow on urban freeway would cause an increase of more than $300 \%$ in the number of conflicts and a decline in traffic safety levels as the PR of CAVs increases from $10 \%$ to $50 \%$. The results indicate that the arrangement of managed lanes for CAVs rises in traffic safety as the PR of CAVs is in the range of $10 \%$ to $50 \%$ compared with allowing CAVs lane-changing. As the PR of CAVs rises above $70 \%$, the safety level rises up again without lane management of CAVs. The reason for the traffic safety level decline may be the driving behavior difference between HVs and CAVs, and the difference may cause interference in vehicle operating and lead to conflicts. The results also indicate that setting managed lanes for CAVs could help improve traffic safety at the freeway crash hotspot. With a better perception of the surrounding conditions and information sharing, CAVs running at the same lane could keep a short head spacing and form a fleet.

Because only one set of traffic flow data was used in this study, the impact of CAVs on traffic safety may not be completely revealed. In the further study, more road situations with different traffic flow shall be considered, and the function of more detailed lane management shall be discussed.

\section{Discussion}

The impact of CAVs on traffic safety would change along with the change of PR of CAVs [11]. The number of potential conflicts increases with the increase of PR of CAVs, with the PR under 50\%. The SD of acceleration also increases, which indicates the decline of traffic safety levels. When the PR of CAVs is between $50 \%$ and $80 \%$, the number of potential conflicts, acceleration distribution, and velocity difference distribution barely change with PRs increasing. CAVs and HVs form a stable traffic flow state. As the PR of CAVs is beyond $80 \%$, the number of potential conflicts and standard deviation of acceleration decrease with an increase in the PR of CAVs. This indicates the improvement of traffic level compared with the situation when the penetration is $50 \%$. But the number of potential conflicts is still higher with the PR of CAVs at $90 \%$ compared with $10 \%$.
After constraining CAVs not to change lanes, the number of potential conflicts and SD of acceleration decrease with an increase in the PR of CAVs, which indicates the improvement of traffic safety level.

Though simulation is relatively cheap and easy to conduct, the reliability and precision of simulation cannot fully reveal the actual rule of CAV field-driving behavior. For further investigation of the influence of CAVs on traffic safety, a real-road CAV test is essential. Two traffic management strategies were proposed in this research, which were no managed lane and managed lane for CAVs. A more specific and customized management strategy could improve traffic safety levels better. The PR mentioned in this research is the proportion rate of CAVs in the process of vehicle input before the simulation. The input proportion rate cannot reflect the $\mathrm{PR}$ in the traffic operating process accurately. For a more accurate investigation of the impact of the mixed condition of CAVs and HVs on traffic safety, controlling the PR during the simulation is essential.

For further research, calibrating the CAV control algorithms is necessary, which could help improve the accuracy of microsimulation and improve the reliability of the research. Experiments with real CAVs could reflect the driving behaviors directly, and traffic safety levels could be evaluated by analyzing the data collected in the experiment. More experiment scenarios could be designed, and a more concrete traffic management strategy should help the traffic flow operating more smoothly.

\section{Data Availability}

The data indicated in the findings have not been made available due to data privacy.

\section{Conflicts of Interest}

The authors declare that there are no conflicts of interest regarding the publication of this paper.

\section{Acknowledgments}

This research was supported by the National Key Research and Development Program of China (2019YFB1600800), National Natural Science Foundation of China (52072289 and 51775396), and Independent Innovation Foundation of Wuhan University of Technology: Risk Assessment and Prediction System of New Energy Vehicle Operation Safety Based on Terminal-Network-Cloud Integration (2020-zy092).

\section{References}

[1] A. D. Tibljas, T. Giuffre, S. Surdonja, and S. Trubia, "Introduction of autonomous vehicles: roundabouts design and safety performance evaluation," Sustainability, vol. 10, no. 4, p. 1060, 2018.

[2] J. Rios-Torres and A. A. Malikopoulos, "A survey on the coordination of connected and automated vehicles at intersections and merging at highway on-ramps," IEEE 
Transactions on Intelligent Transportation Systems, vol. 18, no. 5, pp. 1066-1077, 2017.

[3] L. Wang, M. Abdel-Aty, W. Ma, J. Hu, and H. Zhong, "Quasivehicle-trajectory-based real-time safety analysis for expressways," Transportation Research Part C: Emerging Technologies, vol. 103, pp. 30-38, 2019.

[4] S. C. Calvert, W. J. Schakel, and J. W. C. van Lint, "Will automated vehicles negatively impact traffic flow?," Journal of Advanced Transportation, vol. 2017, Article ID 3082781, 17 pages, 2017.

[5] A. Mirheli, L. Hajibabai, and A. Hajbabaie, "Development of a signal-head-free intersection control logic in a fully connected and autonomous vehicle environment," Transportation Research Part C: Emerging Technologies, vol. 92, pp. 412-425, 2018.

[6] A. Mirheli, M. Tajalli, L. Hajibabai, and A. Hajbabaie, "A consensus-based distributed trajectory control in a signal-free intersection," Transportation Research Part C: Emerging Technologies, vol. 100, pp. 161-176, 2019.

[7] M. Karimi, C. Roncoli, C. Alecsandru, and M. Papageorgiou, "Cooperative merging control via trajectory optimization in mixed vehicular traffic," Transportation Research Part C-Emerging Technologies, vol. 116, Article ID 102663, 2020.

[8] A. Talebpour and H. S. Mahmassani, "Influence of connected and autonomous vehicles on traffic flow stability and throughput," Transportation Research Part C: Emerging Technologies, vol. 71, pp. 143-163, 2016.

[9] J. I. Ge, S. S. Avedisov, C. R. He, W. B. Qin, M. Sadeghpour, and G. Orosz, "Experimental validation of connected automated vehicle design among human-driven vehicles," Transportation Research Part C: Emerging Technologies, vol. 91, pp. 335-352, 2018.

[10] L. Ye and T. Yamamoto, "Evaluating the impact of connected and autonomous vehicles on traffic safety," Physica A: Statistical Mechanics and Its Applications, vol. 526, Article ID 121009, 2019.

[11] N. Virdi, H. Grzybowska, S. T. Waller, and V. Dixit, "A safety assessment of mixed fleets with connected and autonomous vehicles using the surrogate safety assessment module," Accident Analysis \& Prevention, vol. 131, pp. 95-111, 2019.

[12] A. Papadoulis, M. Quddus, and M. Imprialou, "Evaluating the safety impact of connected and autonomous vehicles on motorways," Accident Analysis \& Prevention, vol. 124, pp. 12-22, 2019.

[13] L. Wang, H. Zhong, W. J. Ma, M. Abdel-Aty, and J. Park, "How many crashes can connected vehicle and automated vehicle technologies prevent: a meta-analysis," Accident Analysis and Prevention, vol. 136, Article ID 105299, 2020.

[14] A. Sinha, S. Chand, K. P. Wijayaratna, N. Virdi, and V. Dixit, "Crash severity and rate evaluation of conventional vehicles in mixed fleets with connected and automated vehicles," Procedia Computer Science, vol. 170, pp. 688-695, 2020.

[15] A. Sinha, S. Chand, K. P. Wijayaratna, N. Virdi, and V. Dixit, "Comprehensive safety assessment in mixed fleets with connected and automated vehicles: a crash severity and rate evaluation of conventional vehicles," Accident Analysis and Prevention, vol. 142, Article ID 105567, 2020.

[16] L. Cui, J. Hu, B. B. Park, and P. Bujanovic, "Development of a simulation platform for safety impact analysis considering vehicle dynamics, sensor errors, and communication latencies: assessing cooperative adaptive cruise control under cyber attack," Transportation Research Part C: Emerging Technologies, vol. 97, pp. 1-22, 2018.
[17] C. Wang, C. Xu, J. Xia, Z. Qian, and L. Lu, “A combined use of microscopic traffic simulation and extreme value methods for traffic safety evaluation," Transportation Research Part C: Emerging Technologies, vol. 90, pp. 281-291, 2018.

[18] M. Seraj, J. Li, and Z. Qiu, "Modeling microscopic car-following strategy of mixed traffic to identify optimal platoon configurations for multiobjective decision-making," Journal of Advanced Transportation, vol. 2018, Article ID 7835010, 15 pages, 2018.

[19] S. Gong, J. Shen, and L. Du, "Constrained optimization and distributed computation based car following control of a connected and autonomous vehicle platoon," Transportation Research Part B: Methodological, vol. 94, pp. 314-334, 2016.

[20] Z. H. Khattak, B. L. Smith, H. Park, and M. D. Fontaine, "Cooperative lane control application for fully connected and automated vehicles at multilane freeways," Transportation Research Part C: Emerging Technologies, vol. 111, pp. 294-317, 2020.

[21] Z. Zhong, E. E. Lee, M. Nejad, and J. Lee, "Influence of CAV clustering strategies on mixed traffic flow characteristics: an analysis of vehicle trajectory data," Transportation Research Part C: Emerging Technologies, vol. 115, Article ID 102611, 2020.

[22] R. Niroumand, M. Tajalli, L. Hajibabai, and A. Hajbabaie, "Joint optimization of vehicle-group trajectory and signal timing: introducing the white phase for mixed-autonomy traffic stream," Transportation Research Part C: Emerging Technologies, vol. 116, Article ID 102659, 2020.

[23] Y. Guo, M. Essa, T. Sayed, M. M. Haque, and S. Washington, "A comparison between simulated and field-measured conflicts for safety assessment of signalized intersections in Australia," Transportation Research Part C: Emerging Technologies, vol. 101, pp. 96-110, 2019.

[24] J. So, G. Dedes, B. B. Park, S. HosseinyAlamdary, and D. Grejner-Brzezinsk, "Development and evaluation of an enhanced surrogate safety assessment framework," Transportation Research Part C: Emerging Technologies, vol. 50, pp. 51-67, 2015.

[25] J. Lee, B. Park, K. Malakorn, and J. So, "Sustainability assessments of cooperative vehicle intersection control at an urban corridor," Transportation Research Part C: Emerging Technologies, vol. 32, pp. 193-206, 2013.

[26] O. Giuffrè, A. Granà, M. L. Tumminello et al., "Evaluation of roundabout safety performance through surrogate safety measures from microsimulation," Journal of Advanced Transportation, vol. 2018, Article ID 4915970, 14 pages, 2018.

[27] A. Okabe, T. Satoh, and K. Sugihara, "A kernel density estimation method for networks, its computational method and a GIS-based tool," International Journal of Geographical Information Science, vol. 23, no. 1, pp. 7-32, 2009.

[28] M. S. Rahman, M. Abdel-Aty, J. Lee, and M. H. Rahman, "Safety benefits of arterials' crash risk under connected and automated vehicles," Transportation Research Part C: Emerging Technologies, vol. 100, pp. 354-371, 2019.

[29] H. Jiang, J. Hu, S. An, M. Wang, and B. B. Park, "Eco approaching at an isolated signalized intersection under partially connected and automated vehicles environment," Transportation Research Part C: Emerging Technologies, vol. 79, pp. 290-307, 2017.

[30] M. Zhu, X. Wang, A. Tarko, and S. E. Fang, "Modeling carfollowing behavior on urban expressways in Shanghai: a naturalistic driving study," Transportation Research Part C: Emerging Technologies, vol. 93, pp. 425-445, 2018. 
[31] M. Treiber, A. Hennecke, and D. Helbing, "Congested traffic states in empirical observations and microscopic simulations," Physical Review E, vol. 62, no. 2, pp. 1805-1824, 2000.

[32] M. S. Rahman, M. Abdel-Aty, L. Wang, and J. Lee, "Understanding the highway safety benefits of different approaches of connected vehicles in reduced visibility conditions," Transportation Research Record: Journal of the Transportation Research Board, vol. 2672, no. 19, pp. 91-101, 2018.

[33] A. Talebpour, H. S. Mahmassani, and F. E. Bustamante, "Modeling driver behavior in a connected environment: integrated microscopic simulation of traffic and mobile wireless telecommunication systems," Transportation Research Record: Journal of the Transportation Research Board, vol. 2560, no. 1, pp. 75-86, 2016.

[34] D. Gettman, L. Pu, T. Sayed, and S. G. Shelby, "Surrogate safety assessment model and validation," Final Report, Turner-Fairbank Highway Research Center, McLean, VA, USA, 2008.

[35] M. M. Morando, Q. Y. Tian, L. T. Truong, and H. L. Vu, "Studying the safety impact of autonomous vehicles using simulation-based surrogate safety measures," Journal of Advanced Transportation, vol. 2018, Article ID 6135183, 11 pages, 2018.

[36] X. Y. Cai, C. L. Lei, B. Peng, X. Y. Tang, and Z. G. Gao, "Road traffic safety risk estimation method based on vehicle onboard diagnostic data," Journal of Advanced Transportation, vol. 2020, Article ID 3024101, 13 pages, 2020. 